\title{
A Spatio-temporal Methodology for Real-time Biosurveillance
}

\author{
(to appear in Quality Engineering) \\ Ronald D. Fricker, Jr.* \\ and \\ Joseph T. Chang ${ }^{\dagger}$
}

March 31, 2008

\begin{abstract}
In this paper we introduce a new spatio-temporal methodology for biosurveillance entitled the Repeated Two-sample Rank (RTR) procedure. It is designed to sequentially incorporate information from individual observations and thus can operate on data in real-time as it arrives into an automated biosurveillance system. In addition, upon a signal of a possible outbreak, the methodology suggests a way to graphically indicate the likely outbreak location, and the output can subsequently be used to track the spread of an outbreak. Thus, the methodology can be used for both early event detection and situational awareness in automated biosurveillance systems.
\end{abstract}

KEYWORDS: Biosurveillance, syndromic surveillance, bioterrorism, public health, kernel density estimation, Kolmogorov-Smirnov statistic.

\section{Introduction}

Biosurveillance is the regular collection, analysis, and interpretation of indicators of diseases and disease outbreaks by public health organizations. In the past decade, the focus of biosurveillance has expanded from monitoring naturally occurring diseases to also include maliciously introduced diseases in the form of bioterrorism. The biosurveillance problem is inherently spatio-temporal since a public health practitioner needs to know both when and where an outbreak is occurring. Syndromic surveillance is "the ongoing, systematic collection, analysis, interpretation, and application

\footnotetext{
* Naval Postgraduate School, Operations Research Department, Monterey, CA

${ }^{\dagger}$ Yale University, Department of Statistics, New Haven, CT
} 
of real-time (or near-real-time) indicators of diseases and outbreaks that allow for their detection before public health authorities would otherwise note them" (Sosin, 2003).

Since its inception, syndromic surveillance has mainly focused on early event detection: gathering and analyzing data in advance of diagnostic case confirmation to give early warning of a possible outbreak. Such early event detection is not supposed to provide a definitive determination that an outbreak is occurring. Rather, it is supposed to signal that an outbreak may be occurring, indicating a need for further evidence or triggering an investigation by public health officials.

As discussed in Fricker and Rolka (2006), the focus of biosurveillance has been expanded to include both early event detection and situational awareness. Situational awareness is the real-time analysis and display of health data to monitor the location, magnitude, and spread of an outbreak. As Bravata et al. (2004) said, “...an essential component of preparations for illnesses and syndromes potentially related to bioterrorism includes the deployment of surveillance systems that can rapidly detect and monitor [emphasis added] the course of an outbreak and thus minimize associated morbidity and mortality."

The CDC and many state and local health departments around the United States are actively developing and fielding biosurveillance systems, such as the BioSense system (www.cdc.gov/biosense) and the Early Abberation and Reporting System (EARS) (www.bt.cdc.gov/surveillance/ears/) at the Centers for Disease Control and Prevention (CDC), and the Electronic Surveillance System for the Early Notification of Community-based Epidemics (ESSENCE) (www.geis.fhp.osd.mil/ GEIS/SurveillanceActivities/ESSENCE/ESSENCE.asp) by the Department of Defense. Sosin (2005) states that approximately 100 state and local health jurisdictions were conducting some form of syndromic surveillance in 2003. In 2004, Bravata et al. (2004) conducted a systematic review of the publicly available literature and various websites from which they identified 115 biosurveillance systems.

\section{$1.1 \quad$ Related Literature}

Most syndromic surveillance systems attempt to detect disease outbreaks using variants of the standard univariate statistical process control (SPC) methods: Shewhart, CUSUM, and/or EWMA. Woodall (2006) provides a comprehensive overview of the application of SPC to health surveillance. Montgomery (2001) is an excellent introduction to these methods in an industrial setting. Fricker (2007), Fricker and Rolka (2006), Shmueli and Fienberg (2006), and Shmueli (2006) give a review of these and other methods used in and applicable to biosurveillance. 
Spatio-temporal methods are less common and used less frequently in syndromic surveillance systems. These methods include Kleinman et al. (2004) and Lazarus et al. (2002) who proposed a generalized linear mixed model to simultaneously monitor disease counts over time in a region divided into smaller sub-areas (zip codes). Their method is statistically attractive because it uses information across the entire region while appropriately adjusting for the smaller areas. As described in Kleinman, et al. (2004), there are two forms of the model depending on whether individual data and covariates are available versus aggregated counts and covariates by zip code.

The most commonly used spatial method is the scan statistic, particularly as implemented in the SaTScan software (www.satscan.org). Originally developed to retrospectively identify disease clusters (see Kulldorff, 1997), the method is now regularly used prospectively in electronic biosurveillance systems (see Kulldorff, 2001). For example, it was used as part of a drop-in syndromic surveillance system in New York City after the 9/11 attack (Ackelsberg et al., 2002). Though widely used, some aspects of the prospective application of the SaTScan methodology have been questioned, particularly the use of recurrence intervals and performance comparisons between SaTScan and other methods. See Woodall et al. (2007) for further details.

Other spatio-temporal approaches include Sonesson (2007) who applies a CUSUM methodology to scan statistics and Rogerson and Yamada (2004) who apply CUSUM methods to the spatial distribution of cases. Diggle et al. (2004) use a spatio-temporal Cox point process methodology based on the counts in subregions. Olson et al. (2005) and Forsberg et al. (2006) assess possible disease clusters using M-statistics based on the distribution of pairwise distances between cases. See Lawson and Kleinman (2005) for additional exposition and methods, and Mandl et al. (2004) for further discussion of spatial and spatio-temporal modeling issues. For spatial methods with application to more traditional public health data and problems, see Waller and Gotway (2004).

\subsection{The Problem and One Solution}

The purpose of biosurveillance is to detect unusual patterns (generally increases) in the incidence of disease or, in the case of syndromic surveillance, unusual patterns in leading indicators of disease. These patterns may be clusters, much like we might think about the emergence of a cluster of cancer, but they may also be other patterns reflecting some other type of increase in disease incidence.

In the context of early event detection, one purpose of a biosurveillance methodology is to signal the suspected pattern as quickly as possible within the constraint of a tolerable false signal rate. In the context of situational awareness, a biosurveillance methodology should also provide on-going 
information about the extent and spread of a disease over time. In industrial quality control terminology, early event detection is akin to detecting the shift in a quality characteristic using a statistical process control methodology while situational awareness is akin to continuous process monitoring in order to understand how to manage a process.

Timeliness of detection in biosurveillance is of particular importance. Timeliness can be achieved either through the development of methods that are more sensitive and/or that can incorporate information and signal in real-time. All of the existing methods of which we are aware, including those described in the previous section, use data aggregated in either space and/or time, usually on a daily basis. This aggregation limits the timeliness of the procedures to, at best, a daily signal. In biosurveillance, assuming the real-time delivery of data at the individual observation level, the ideal method should incorporate the information from each observation as it occurs and signal just as soon as there is sufficient evidence of an anomaly.

Given a signal, it is then important to provide public health practitioners with some indication about where the outbreak is occurring and should be able to then provide on-going plots of the spread. Purely temporal methods by definition cannot do this, so that upon a signal public health practitioners then have to sift through the data looking for the cause of the signal. Spatio-temporal methods often do provide an indication of the spatial location for the signal, though they may be more or less suited to continuing to provide continuing information about the spread of the disease. A methodology that can be readily adapted for real-time biosurveillance is the Repeated Two-sample Rank (RTR) procedure of Fricker (1997). The method is designed to incorporate the information from individual observations and can be used to identify the location of anomaly. In addition, the approach used by the RTR procedure can naturally be used to display the spread of a disease over time once a signal has been raised. The RTR procedure uses kernel density estimation (KDE) to calculate the density heights of a set of historical observations, representing the normal incidence of a disease, and a set of new data, reflecting the current state. Disease outbreaks are identified by comparing the historical data and new data density height distributions. The new set of data is constantly updated and tested as observations arrive. In addition, comparisons between a kernel density estimate for the historical data and one for the new data provide information about where the outbreak occurs and how it spreads. 


\subsection{Outline of this Paper}

The paper is organized as follows. In Section 2 we describe the RTR procedure. In Section 3 we describe how to apply the RTR procedure to the biosurveillance problem and demonstrate its performance using some simulated disease outbreaks. In Section 4 we discuss our results and provide some conclusions.

\section{Repeated Two-sample Rank Procedure}

The Repeated Two-sample Rank (RTR) procedure was introduced by Fricker (1997). Consider a sequence of bivariate observations $\mathbf{X}_{i}=\left\{X_{1, i}, X_{2, i}\right\}$. Think of each $\mathbf{X}_{i}$ as the location of one occurrence of a disease. For example, it might be the latitude and longitude of the home address of each individual that presents to a hospital emergency room with a particular syndrome or of each individual diagnosed with a particular disease. The goals are to: (1) detect quickly when the distribution of disease incidence changes, and (2) when such a change is signalled, provide information about the location or locations of increased disease incidence.

Assume $\mathbf{X}_{1}, \ldots, \mathbf{X}_{\tau-1}$ are independent and identically distributed (iid) according to some density $f_{0}$ that corresponds to the natural state of disease incidence and $\mathbf{X}_{\tau}, \mathbf{X}_{\tau+1}, \ldots$ are iid according to anther density $f_{1}$ which corresponds to an increase in disease incidence in some portion of the region being monitored. The densities $f_{0}$ and $f_{1}$ are unknown. The change point $\tau$ is the time when the process switches from the normal background disease incidence ("non-outbreak") state to an elevated disease incidence ("outbreak") state.

Assume that a historical sample of data $\mathbf{Y}_{1}, \ldots, \mathbf{Y}_{N}$ is available. The disease incidence is assumed to have been in a non-outbreak state throughout the historical sample, so that the historical observations are distributed according to $f_{0}$. The historical sample is followed by new data $\mathbf{X}_{1}, \mathbf{X}_{2}, \ldots$, whose density may change from $f_{0}$ to another density at some unknown time. For notational convenience, define $\mathbf{X}_{i}=\mathbf{Y}_{N+i}$ for $i \leq 0$. Also consider a set of the $w+1$ most recent data points $\mathbf{X}_{n-w}, \ldots, \mathbf{X}_{n}$ which will be used to decide whether or not the process is in an outbreak state at the time when observation $n$ arrives.

The RTR procedure uses a kernel estimate $\hat{f}_{n}$ formed from the historical sample data and the new data, defined as follows. Given a kernel function $k$ (which is usually a density on $\mathbb{R}^{2}$ ) and a 
bandwidth $h>0$,

$$
\hat{f}_{n}(\mathbf{x})= \begin{cases}\frac{1}{N+n} \sum_{i=1-N}^{n} k_{h}\left(\mathbf{x}, \mathbf{X}_{i}\right), & n<w+1 \\ \frac{1}{N+w+1} \sum_{i=n-w-N-1}^{n} k_{h}\left(\mathbf{x}, \mathbf{X}_{i}\right), & n \geq w+1\end{cases}
$$

where $k_{h}\left(\mathbf{x}, \mathbf{X}_{i}\right)=h^{-2} k\left[\left(x_{1}-X_{1, i}, x_{2}-X_{2, i}\right) / h\right]$, and where $\mathbf{x}=\left\{x_{1}, x_{2}\right\}$ is the point in the plane at which the function is evaluated. The reason for the two expressions in Equation (1) is to allow the RTR to begin testing new data starting with the first observation and not have to wait until all of the first $w+1$ new observations have arrived. The density estimate $\hat{f}_{n}$ is evaluated at each historical point and each data point in the new data, obtaining the values

$$
\underbrace{\hat{f}_{n}\left(\mathbf{X}_{1-N}\right), \ldots, \hat{f}_{n}\left(\mathbf{X}_{0}\right)}_{\text {historical observations }}, \underbrace{\hat{f}_{n}\left(\mathbf{X}_{1}\right), \ldots, \hat{f}_{n}\left(\mathbf{X}_{n}\right)}_{\text {new observations }}
$$

when $n<w+1$ or

$$
\underbrace{\hat{f}_{n}\left(\mathbf{X}_{n-w-N-1}\right), \ldots, \hat{f}_{n}\left(\mathbf{X}_{n-w-1}\right)}_{\text {historical observations }}, \underbrace{\hat{f}_{n}\left(\mathbf{X}_{n-w}\right), \ldots, \hat{f}_{n}\left(\mathbf{X}_{n}\right)}_{\text {new observations }}
$$

when $n \geq w+1$.

If the process is still in a non-outbreak state at the time when observation $n$ occurs, so that the $\mathbf{X}_{i}$ are iid then, via a small generalization of Theorem 11.2.3 of Randles and Wolfe (1979, page 356), the estimated density heights within (2) and within (3) are exchangeable, so that all rankings of them are equally likely. Given this, the procedure performs a hypothesis test on the ranks at each time when a new observation arrives, and signals at the first time the test rejects the hypothesis that the ranks of the estimated density heights of the new sample of data are uniformly distributed among the ranks of the density heights of the historical sample.

The hypothesis test used here is a Kolmogorov-Smirnov test. For notational convenience, assume $n \geq w+1$ and let $\hat{J}_{n}$ denote the empirical distribution function of the density heights $\hat{f}_{n}\left(\mathbf{X}_{n-w}\right), \ldots, \hat{f}_{n}\left(\mathbf{X}_{n}\right)$ for the new data, defined by

$$
\hat{J}_{n}(z)=\frac{1}{w+1} \sum_{i=n-w}^{n} I\left\{\hat{f}_{n}\left(\mathbf{X}_{i}\right) \leq z\right\}
$$

where $I$ denotes the indicator function. Similarly, for the historical sample, define

$$
\hat{H}_{N}(z)=\frac{1}{N} \sum_{i=n-w-N-1}^{n-w-1} I\left\{\hat{f}_{n}\left(\mathbf{X}_{i}\right) \leq z\right\}
$$


The Kolmogorov-Smirnov statistic at the time when observation $n$ arrives is

$$
S_{n}=\max _{z}\left(\hat{J}_{n}(z)-\hat{H}_{N}(z)\right),
$$

which is the largest positive pointwise distance from the empirical distribution in (5) to the empirical distribution in (4). Given a threshold $c$, the procedure stops and signals at the first time $t$ that $S_{n}$ is greater than $c: t=\min \left\{n: S_{n}>c\right\}$.

The use of a "one-sided" Kolmogorov-Smirnov statistic in Equation (6) implicitly assumes we are looking for outbreaks in regions that have lower historical levels of disease incidence. If the goal is to monitor for outbreaks throughout the region then one should use the usual Kolmogorov-Smirnov statistic

$$
S_{n}=\max _{z}\left|\hat{J}_{n}(z)-\hat{H}_{N}(z)\right|
$$

A false signal occurs if the procedure stops but no outbreak has occurred, that is, $t<\tau$. The threshold is selected so that, under the hypothesis that an outbreak never occurs, the average time to (false) signal (ATS) is suitably large.

In summary, the RTR procedure proceeds as follows.

1. Choose a historical sample size $N$, a new sample size $w+1$ (where $N \gg w+1$ ), and set a threshold $c$ to achieve a desired ATS.

2. Collect an historical sample of data points during which the background disease incidence is in a non-outbreak state and set $n=1$.

3. Using $w+1$ of the most recent data points, calculate the estimated density heights for the historical sample and the new data using Equation (1).

4. Calculate the Kolmogorov-Smirnov statistic $S_{n}$ according to Equation (6):

- If $S_{n} \geq c$, stop and signal that an outbreak may be occurring.

- If $S_{n}<c$, when a new observation arrives, increment $n$, update the historical and new data sets, and go to step 3 and repeat.

\section{Applying the RTR Procedure to Biosurveillance}

What makes the RTR procedure unique among spatio-temporal biosurveillance methods is that it is designed to incorporate information from each observation, one at a time, as they arrive into a 
biosurveillance system. However, to apply the RTR procedure as a (near) real-time biosurveillance tool the data must: (1) come in (near) real-time and, (2) contain location information on each individual.

Now, while data that arrives more slowly or perhaps aggregated (say, by day) does not preclude the use of the RTR procedure, the speed with which data arrives will drive how timely its resulting signals will be. For example, we will show some simulations in which the procedure produces a signal during a large outbreak on the first day. If the data arrives in real time, then the procedure will signal part way through day 1 just as soon as sufficient evidence accumulates that something unusual is happening. If, for that same data, the procedure must wait until the end of day 1 or day 2 to get an aggregate "dump" of day 1's data, then the signal will be correspondingly delayed.

Location information is critical since the RTR procedure constructs estimated densities of the spatial distribution of disease incidence. There are some significant challenges are inherent in using such location data. For example, should the location of an individual correspond to, say, a home address or a work address? Similarly, what is the appropriate way to determine the location of transient individuals, such as business travelers? For the purposes of this paper we will not seek to answer these important issues but simply assume that location information is available for each individual according to a clear, consistent, and medically appropriate definition.

\subsection{Setting RTR Parameters}

Given that the requisite data is available, implementation of the RTR procedure requires choosing and setting various parameters. In particular, one must choose an historical sample size $N$ and which data to include in the historical sample, the new data sample size $w+1$, a kernel distribution, a bandwidth $h$, and a threshold $c$.

Setting the specific size of $N$ and $w$ is a subjective judgement based on the typical number of daily observations and how far back in time data is still appropriate for incorporation into the historical distribution. That is, in the historical distribution more data is better so long as the data is not so old that it no longer reflects current disease incidence patterns. However, given that trends are often present, it is usually prudent to limit the amount of historical data to only that necessary to estimate the historical distribution well.

In the RTR procedure the window size acts like a smoothing parameter. Choosing a value of $w$ that is too small results in vulnerability to noise, and an excessively large value introduces too much 
inertia into the procedure, making quick detection of a change difficult. Hence, it is important to set $w$ sufficiently large so that there are enough observations to reasonably estimate the distribution, but not so many that an outbreak would be masked by a large number of non-outbreak observations.

We think about setting $N$ and $w$ in terms of days of observations. In a situation where there are annual trends in disease incidence, assuming there is a large enough average number of daily observations, using 45 days of historical observations and a window of about 7 days of the most recent observations seems reasonable. So, for example, if the expected number of observations is 30 per day, we set $N=45 \times 30=1,350$ and we might set $w+1=250$ for roughly 7 days of observations at an increased disease incidence rate. If the average number of daily observations is very low, however, then the number of days to include in the historical and new data may need to be larger.

Given the choice of $N$ and $w$, we set the threshold $c$ using the results of Fricker (1997) who, using the Poisson Clumping Heuristic of Aldous (1989), derived a number of approximations for finding the average number of observations $A$ between false signals for a given threshold. Fricker ultimately preferred "Approximation \#1" below based on comparisons with simulation results:

$$
A \approx\left[\left(\frac{6.16 c[c+0.5 /(w+1)]}{1+(w+1) / N}\right) \exp \left\{-2\left(c+\frac{1}{2(w+1)}\right)^{2}\left(\frac{1}{w+1}+\frac{1}{N}\right)^{-1}\right\}\right]^{-1}
$$

However, this approximation is based on the Kolmogorov-Smirnov statistic in Equation (7), while in this problem we are looking for outbreaks in regions that have lower historical levels of disease incidence. Hence we are only interested in the one-sided statistic - Equation (6). That is, here we focus on detecting when the empirical distribution function for the new data contains an unusually large number of small density heights.

Since it is equally likely that differences between the two empirical distributions will occur in one direction as the other, the one-sided test is half as likely to exceed $c$ as the two-sided test, and thus it follows that

$$
A^{\prime} \approx 2 \times A
$$

where $A^{\prime}$ is the approximate average number of observations between false signals for the RTR procedure using a one-sided Kolmogorov-Smirnov statistic for a given threshold $c$.

As with $N$ and $w$, we like to think about setting the threshold in terms of time: the average number of days between false signals. For example, setting $c=0.07754$ (with $N=1,350$ and $w+1=250$ ) in Equation (9) gives $A=900$. Assuming an average of 30 observations per day, this gives an ATS 
of 900 observations divided by 30 observations per day or 30 days between false signals.

In terms of the choice of kernel distribution, we use a simple bivariate normal distribution with no correlation. Fricker (1997) evaluated various alternatives and found that the choice of kernel distribution made little difference in the performance of the RTR procedure.

Finally, the choice of bandwidth $h=\left\{h_{1}, h_{2}\right\}$ can be based on the kernel density estimation literature. Per Bowman and Azzalini (2004), the optimal choice of bandwidth $h_{i}$ is

$$
h_{i}=\sigma_{i}\left(\frac{4}{(p+2) m}\right)^{1 /(p+4)}
$$

where $\sigma_{i}$ is the standard deviation in dimension $i, i=1, \ldots, p$, and $m$ is the number of observations. In this application $p=2$, so the expression reduces to

$$
h_{i}=\sigma_{i}\left(\frac{1}{m}\right)^{1 / 6}
$$

Thus, for the RTR procedure we set $m=N+w+1$, which has the effect of slightly oversmoothing the density estimate early on when $n<w+1$, but which seems to have little effect on performance. Given a signal, we display the differences between the density estimate for the new data and the historical data using $m=w+1$ and $m=N$ for the respective density estimates. More on this in the next section.

\subsection{Simulating Outbreaks}

To illustrate the RTR's performance, we simulated three idealized, but not unrealistic, outbreak scenarios: a localized outbreak that increases linearly, an outbreak that increases quadratically and spreads throughout the population, and an outbreak that sweeps through a region like a contagious disease might. Specifically:

- Scenario \#1: We assume that a hospital is located in the center of a region $(\{0,0\})$. The background disease incidence in the surrounding population occurs according to a bivariate normal distribution centered on the hospital, $N\left(\{0,0\}, \sigma^{2} \mathbf{I}\right)$, where $\mathbf{I}$ is the identity matrix and $\sigma=15$ (miles, say), with an expected number of background cases of 30 per day. The outbreak occurs according to a bivariate normal distribution $N\left(\{20,20\}, d^{2} \mathbf{I}\right)$, where $d$ is the day of the outbreak, with an expected number of outbreak cases of $d$ per day for each day of the outbreak. Thus, the outbreak is centered at $\{20,20\}$, spreading out and growing linearly over time, and, during the outbreak, the expected total number of cases is $30+d$. 
- Scenario \#2: As in Scenario \#1, we assume the background disease incidence in the surrounding population occurs according to $N\left(\{0,0\}, \sigma^{2} \mathbf{I}\right)$ with an expected number of background cases of 30 per day. In this scenario, however, the outbreak occurs according to a bivariate normal distribution $N\left(\{20,20\}, 2.2 d^{2} \mathbf{I}\right)$, where $d$ is the day of the outbreak, with an expected number of outbreak cases of $d^{2}$ per day for each day of the outbreak (so that, during the outbreak, the expected total number of cases is $\left.30+d^{2}\right)$. Thus, the outbreak is centered at $\{20,20\}$, spreading out faster than in Scenario \#1 and growing quadratically in size over time.

- Scenario \#3: The background disease incidence in the surrounding population again occurs according to $N\left(\{0,0\}, \sigma^{2} \mathbf{I}\right)$ with an expected number of background cases of 30 per day. However, in this scenario the outbreak sweeps through the region, perhaps like a contagious disease might pass through. Specifically, the outbreak sweeps through afflicting a strip of the region eight units (say, miles) wide on each day with an expected number of outbreak cases of 64 per day for each day of the outbreak (so that, during the outbreak, the expected total number of cases is $30+64$ ).

Individual realizations of the three scenarios are shown in Figures 1 through 3 for the first 11 days of each outbreak. Day 0 is the day prior to the outbreak.

The first column in each figure shows the distribution of the expected number of cases where the area under the surface for some subregion represents the expected number of cases in that subregion. Of course, in real biosurveillance this distribution is unobserved. As just described, for all three scenarios the background disease incidence follows a simple bivariate normal distribution with an expected number of 30 cases per day. The outbreaks start on day 1 in each figure and show up as an outbreak distribution overlaid on the background disease distribution. The progression of the outbreak distribution can then be followed for the first 11 days.

The second column shows the observations that occurred on each day. On day 0 of each scenario there are about 30 observations generated according to the background disease distribution. Starting on day 1 outbreak observations are intermixed according to each scenario's outbreak type and expected number. On day $d$, the expected number of outbreak observations is $d$ in Scenario \#1, $d^{2}$ in Scenario \#2, and 64 in Scenario \#3.

Finally, the third column shows the contours of the difference between the kernel density estimate for the $N$ historical observations and the kernel density estimate of the $w+1$ new observations calculated as follows. To simplify the notation, assume $n>w+1$ and so define the kernel density 


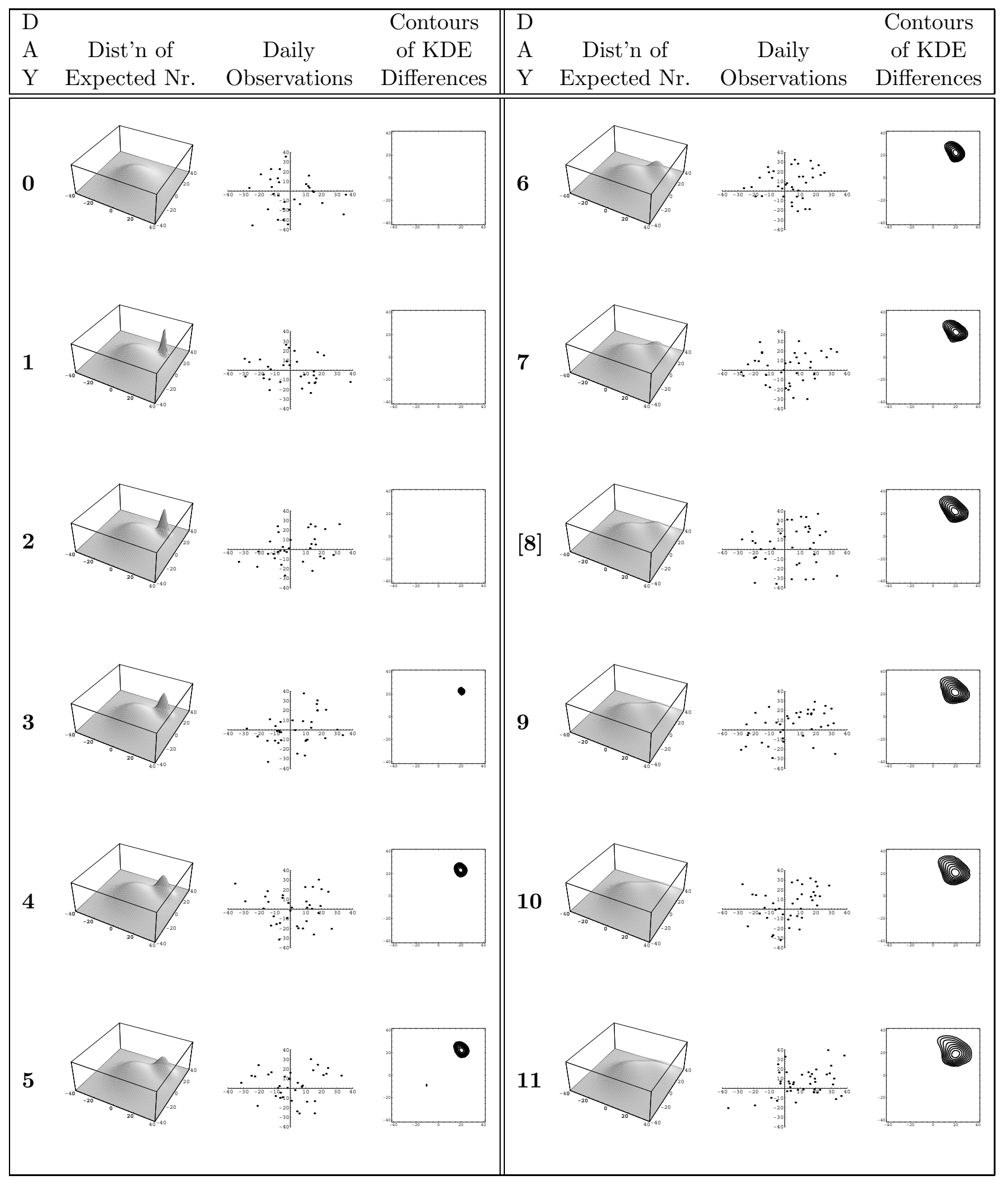

Figure 1: Scenario \#1 begins with a background disease incidence distributed according to a bivariate normal centered at $\{0,0\}$ with an average of 30 observations per day. On day 1 an outbreak begins, centered at $\{20,20\}$ which grows linearly over time and spreads. On day $d$ the expected number of outbreak cases is $d$. 


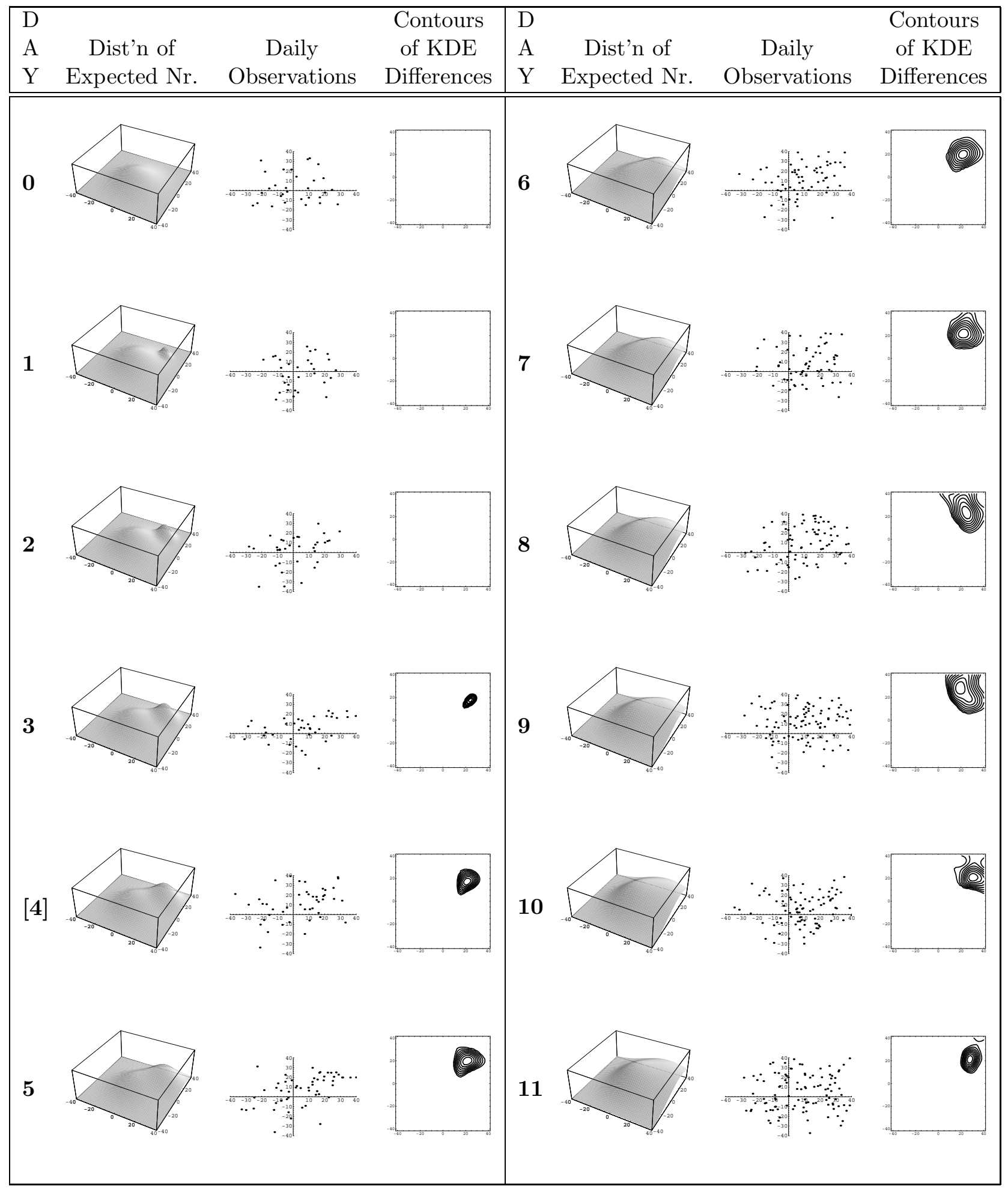

Figure 2: Scenario \#2 also begins with a background disease incidence distributed according to a bivariate normal centered at $\{0,0\}$ with an average of 30 observations per day. On day 1 an outbreak begins, centered at $\{20,20\}$, which grows quadratically over time and spreads faster than the outbreak in Scenario \#1. On day $d$ the expected number of outbreak cases is $d^{2}$. 


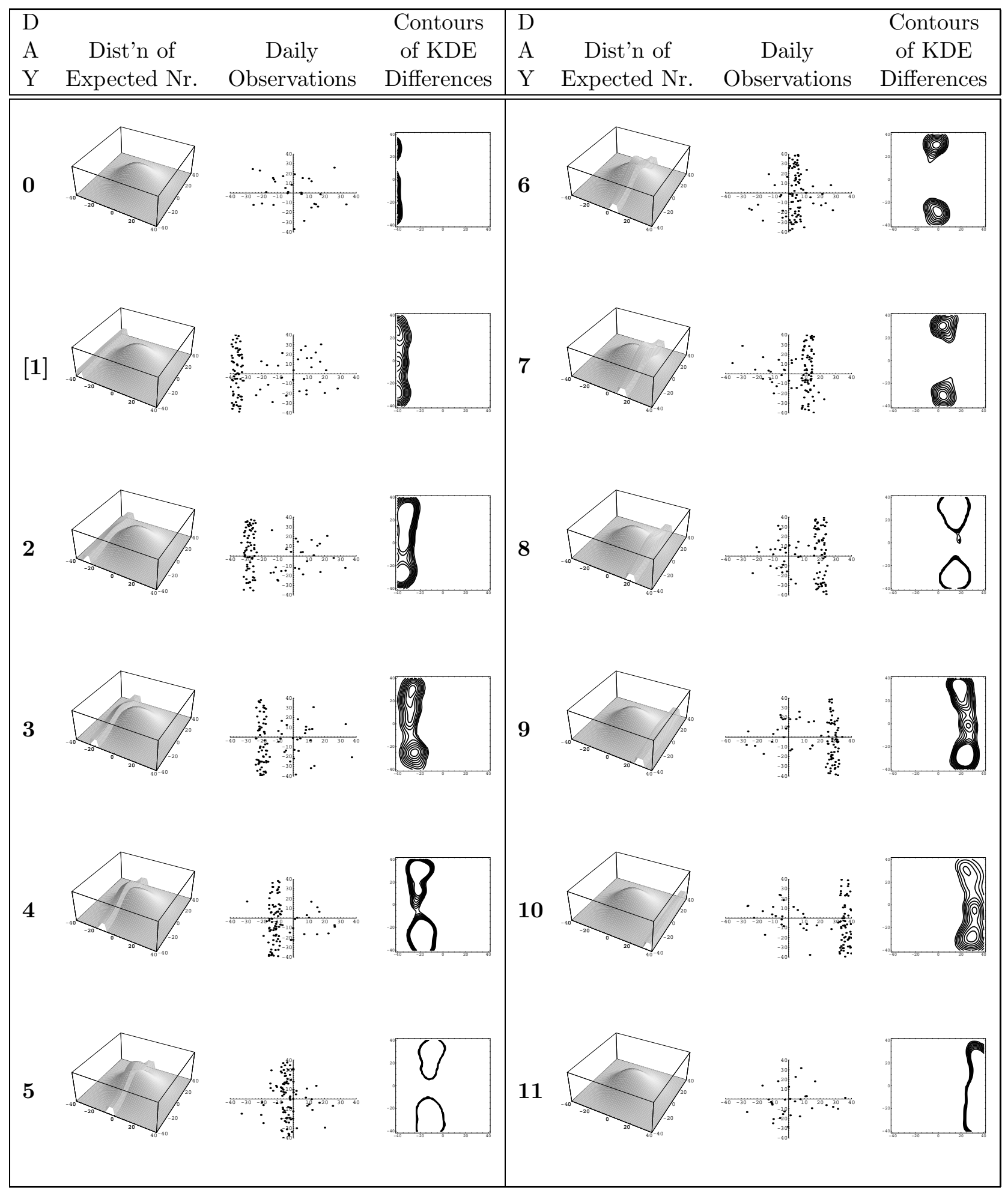

Figure 3: Scenario \#3 begins with a background disease incidence distributed according to a bivariate normal centered at $\{0,0\}$ with an average of 30 observations per day. Starting on day 1, an outbreak sweeps across the region from left to right generating an extra 64 cases per day. 
estimate for the historical data as

$$
\hat{g}_{n}(\mathbf{x})=\frac{1}{N} \sum_{i=n-w-N-1}^{n-w-1} k_{h}\left(\mathbf{x}, \mathbf{X}_{i}\right)
$$

where the kernel function is a bivariate normal and the bandwidth is $h_{i}=\sigma_{i}\left(\frac{1}{N}\right)^{1 / 6}, i=1,2$. Similarly, define the kernel density estimate for the new data as

$$
\hat{h}_{n}(\mathbf{x})=\frac{1}{w+1} \sum_{i=n-w}^{n} k_{h}\left(\mathbf{x}, \mathbf{X}_{i}\right)
$$

where the kernel function is a bivariate normal and the bandwidth is $h_{i}=\sigma_{i}\left(\frac{1}{w+1}\right)^{1 / 6}, i=1,2$. Then plot

$$
\Delta_{n}(\mathbf{x})=\max \left(\delta, \hat{h}_{n}(\mathbf{x})-\hat{g}_{n}(\mathbf{x})\right)
$$

where $\delta$ is a small positive number that helps eliminate excess noise from the plots. For Figures 1 through 3 we used $\delta=0.00011$.

A number of observations arise upon examination of Figures 1 through 3. First, early in Scenarios \#1 and \#2 the scatterplots provide no visual indication that an outbreak is occurring. However, the contours of the kernel density estimate (KDE) differences from Equation (10) correspond nicely to the known outbreak areas. Furthermore, the sequence of the kernel density estimate differences tracks well with the growth of the outbreaks.

That said, it would be erroneous to assume that the contours are sufficient for identifying the outbreaks. This is because it is possible for contours to be visible even when an outbreak is not occurring. To illustrate, see Figure 4 which shows the contour plots for days 0-5 in Scenario \#1 from Figure 1 along with the complete surface plots. Here we see that the differences between the density estimates is noisy and this noise can sometimes show up on the contour plots.

Thus, we supplement a visual inspection of the plots with an analysis of the data using the RTR procedure to signal when a change has occurred. The day on which the RTR signalled when applied to the actual data in each figure is indicated by the day number in brackets: in Figure 1, the RTR signalled on observation 274 on day 8; in Figure 2 the RTR signalled on observation 173 on day 5; and in Figure 3 the RTR signalled on observation 14 on day 1. This latter result indicates the utility of a procedure that operates sequentially on the individual observations. Were the data aggregated by day, then a signal would not have been generated until day 2 , in spite of the fact that by then the outbreak in this case is obvious just by looking at the scatterplot. Instead, the 

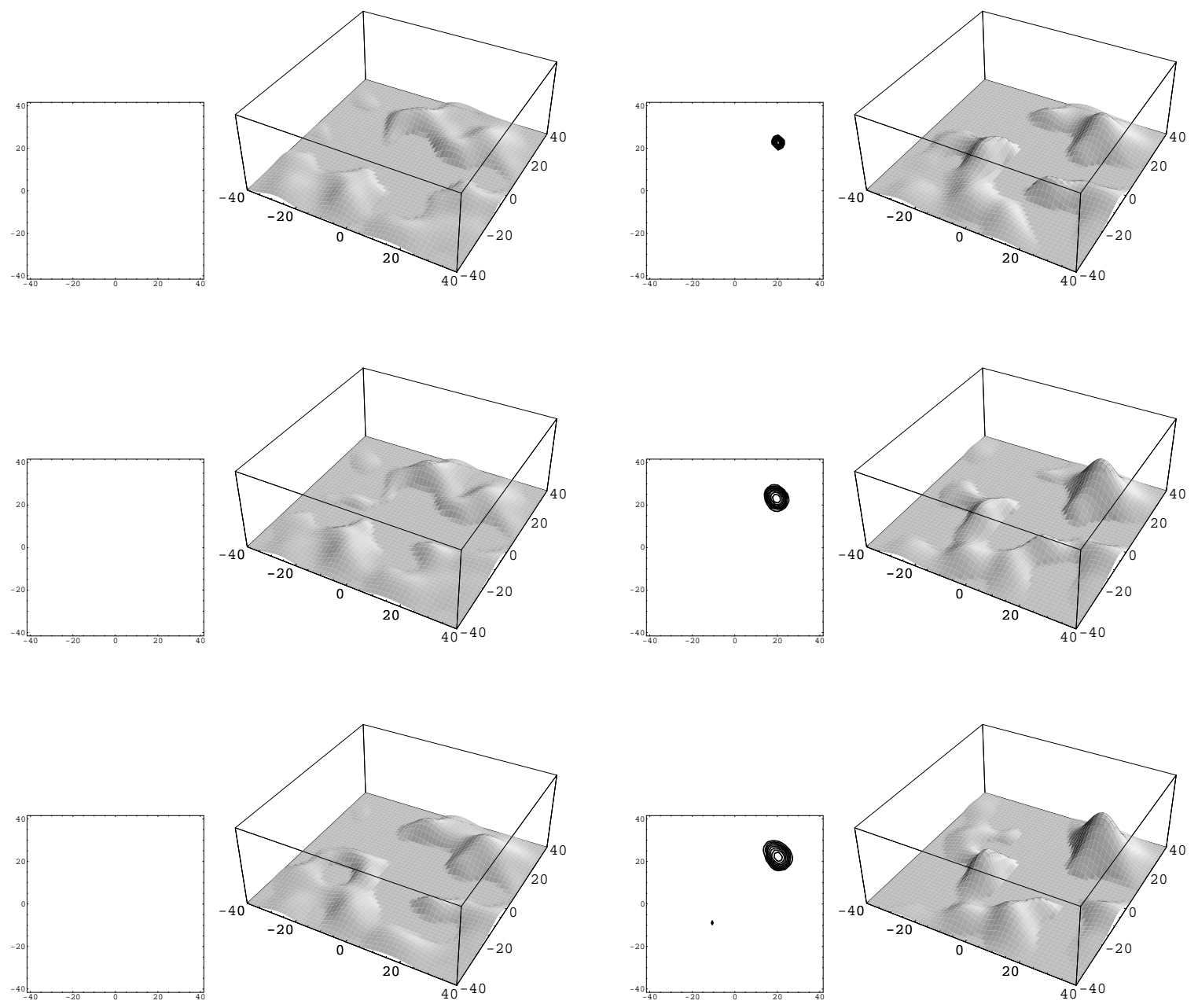

Figure 4: Contour plots and their associated surfaces for days 0-5 in Scenario \#1 from Figure 1. 


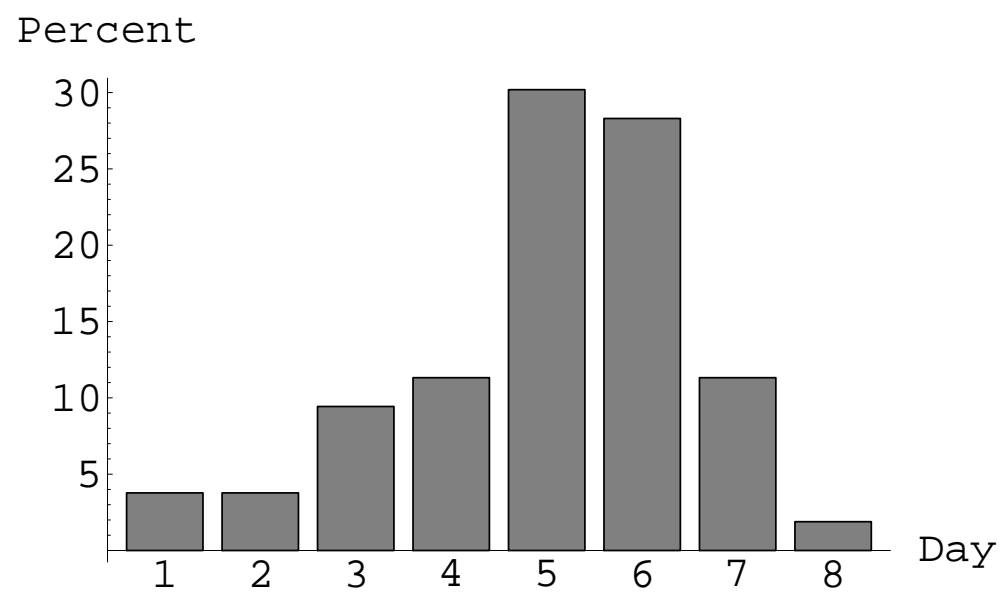

Figure 5: For Scenario \#1, a plot of the percentage of times the RTR stopped on a particular day of the outbreak.

RTR procedure signalled that something unusual seemed to be going on after only 14 observations - roughly 15 percent of the observations that would occur on that day.

Of course, these are only one realization for each of the scenarios and, in fact, the results beg the question as to how the RTR procedure would perform over many trials. As the next section shows, the signal in only 14 observations in Figure 3 was a bit faster than how the RTR would perform on average for Scenario \#3 while the stopping times for the Figures 1 and 2 turn out to be longer than the average for Scenarios \#1 and \#2.

\subsection{Demonstrating the RTR's Performance}

To assess how the RTR procedure performed more generally, we ran it many times on each scenario and recorded the day and the observation number when the RTR signaled. For example, Figure 5 shows that under Scenario \#1 the RTR predominantly signalled on either the 5th or 6th day of the outbreak. Remember that the Scenario \#1 the outbreak increased linearly, so that by the end of the fifth day 15 outbreak observations had been observed on average (in addition to an average of 150 non-outbreak observations). The average number of observations until the RTR signalled was 146 , or roughly in the middle of the 5 th day. 


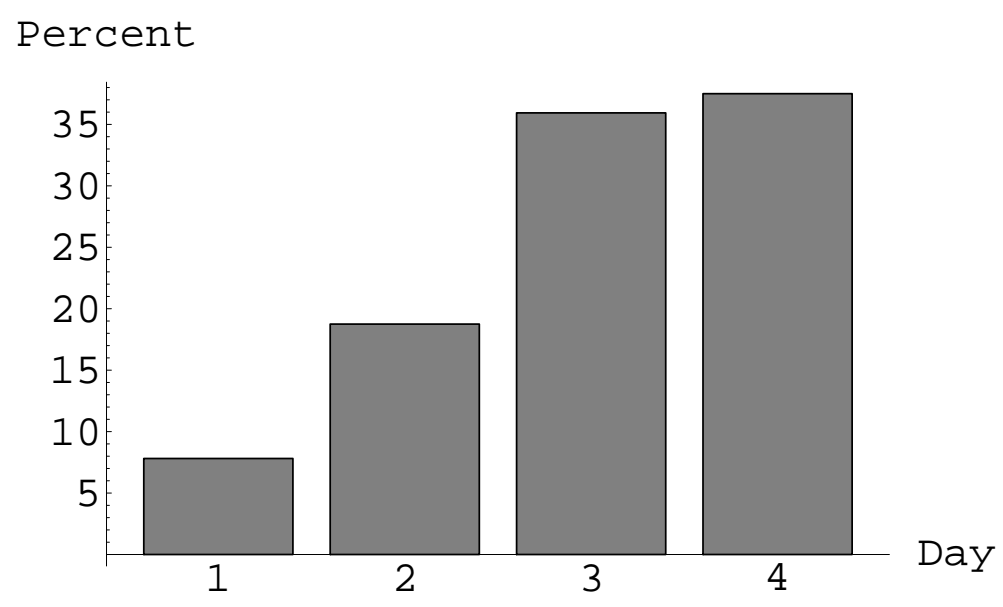

Figure 6: For Scenario \#2, a plot of the percentage of times the RTR stopped on a particular day of the outbreak.

In contrast, Figure 6 shows that under Scenario \#2 the RTR predominantly signalled on the 3rd or 4 th day of the outbreak. This makes sense since under Scenario \#2 the outbreak increased quadratically and so should have been easier to detect than the Scenario \#1 outbreak. In fact, by the end of the third day on average 14 outbreak (and 90 non-outbreak) observations had been observed compared to an average of 15 at the end of the 5th day in Scenario \#1. Under Scenario \#2, the average number of observations until the RTR signalled was 80 , or roughly in the middle of the third day of an outbreak.

Now, under Scenario \#3, the RTR always signalled on the first day. It did so because even just a few observations far out on the periphery of the region would be unusual. Indeed, a simple visual examination of the scatterplots in Figure 3 make it clear that something abnormal is occurring (assuming one knows what the normal pattern looks like). In fact, it took only 23 observations on average until a signal was produced. Given that each day would get, on average 94 observations (64 outbreak and 30 non-outbreak), that means the RTR signalled less than one-third of the way through the first day and after observing about 15 outbreak observations. 


\section{Conclusions}

This paper has demonstrated the application of the Repeated Two-Sample Rank procedure to the problem of biosurveillance and it has shown that methodology supports both goals of syndromic surveillance systems: early event detection and situational awareness. Furthermore, because the RTR procedure is designed to incorporate the information from each individual observation as it sequentially arrives into such a system, the methodology can provide more timely signals than those methods that aggregate data. Indeed, in the simulations we observed that an average of 14 or 15 outbreak observations was sufficient to cause a signal and the RTR was able to synthesize the information from those observations whether they occurred in one day or across many days. In addition, via the use of kernel density estimation, the RTR eliminates the issues faced by other methods which must aggregate data within artificial spatial boundaries (e.g., zip codes). Finally, theoretical results are available to assist the public health practitioner or biosurveillance system designer in choosing the necessary algorithmic parameters such as the kernel bandwidth and the threshold.

The RTR procedure does have some limitations. Most importantly, it is incapable of detecting an increase in disease incidence if the increase is randomly distributed over the region according the background disease incidence (non-outbreak) distribution. If this is of concern then the RTR procedure will have to be augmented with an appropriate temporal method. However, we expect that a disease outbreak or a bioterrorism event is very unlikely to manifest itself in such a fashion. Rather, our sense is that a general increase in disease incidence is likely to be the result of seasonal fluctuations or perhaps some phenomenon related, say, to an aging population. As such, in the context of bioterrorism detection, the RTR's insensitivity to this type of change can be seen as an advantage since it does not have to be adjusted to account for naturally-occurring incidence rate changes the way temporal methods often must be.

The RTR as described in this paper is designed to account for changes in the distribution of background disease. It does this by using a moving window of historical data, for which we arbitrarily chose a window 45 days in length. The idea is that, in biosurveillance, we are monitoring for abrupt departures from recent patterns. The length of this window should be a function both of how quickly the background incidence distribution changes and the rate of the observed data. The key consideration is that the historical data should be of sufficient number to estimate well the non-outbreak distribution and such that the resulting distribution is as current as possible. While Section 2 described the historical sample as a moving window, it need not be so. In particular, 
in biosurveillance settings in which the background disease incidence does not change (or changes very little) over time, it may be preferable to use a fixed historical sample (see Fricker, 1997).

If a moving window of historical data is to be used, there are some practical considerations that must be addressed in the implementation of the methodology related to how to adjust the historical data set once an outbreak is identified. Simply put, it is important to ensure that the historical sample is not contaminated with outbreak data. Such contamination could make it more difficult to detect future outbreaks. See Fricker, Knitt and $\mathrm{Hu}$ (2008) for a discussion of these issues in a related syndromic surveillance context.

Many other variations of the RTR procedure are possible. In this paper, we used density height calculated from a kernel density estimate as the univariate statistic. In work not shown here, we have compared this formulation against variants using data depth and Euclidean distance to nearest neighbor statistics and found kernel density estimation to be preferable based on performance and calculation considerations. We have also compared the use of the Kolmogorov-Smirnov nonparametric test to the chi-squared test. We found the two perform similarly, and our preference for the Kolmogorov-Smirnov test is based on not having to specify "bins." It is also possible to use an adaptive kernel density estimate, which may be preferable when there are subregions in which the background disease incident counts are very low, but we have not explored the performance of such a method in our research.

We conclude by noting that biosurveillance is but one application for the RTR procedure. As described herein, it can be applied to many different types of spatio-temporal change detection, from other types of public health problems, to problems in demography and geography, as well as national security problems such as changes in the employment patterns of improvised explosive devices in Iraq. In addition, though not described here, the RTR procedure can also be used as a purely temporal nonparametric multivariate statistical process control methodology.

Acknowledgments. R. Fricker's research was supported in part by Office of Naval Research grant N0001407WR20172 and in part by funding from the Naval Postgraduate School. 


\section{References}

Ackelsberg, J., Balter, S., Bornschelgel, K., Carubis, E., Cherry, C., Das, D., Fine, A., Karpati, A., Layton, M., Mostashari, F., Nivin, B., Reddy, V., Weiss, D., Hutwagner, L., Seeman, G.M., McQuiston, J., Treadwell, T., and J. Rhodes (2002). Syndromic Surveillance for Bioterrorism Following the Attacks on the World Trade Center - New York City, 2001, Morbidity and Mortality Weekly Report, 51 (Special Issue), Centers for Disease Control and Prevention, pp. 13-15.

Aldous, D. (1989). Probability Approximations via the Poisson Clumping Heuristic, Springer-Verlag, New York, New York.

Bowman, A.W., and A. Azzalini (2004). Applied Smoothing Techniques for Data Analysis: The Kernel Approach with S-Plus Illustrations, Oxford Science Publications, Clarendon Press, Oxford, England.

Bravata, D.M., McDonald, K.M., Smithe, W.M., Rydzak, C., Szeto, H., Buckeridge, D.L., Haberland, C., and D.K. Owens (2004). Systematic review: Surveillance Systems for Early Detection of Bioterrorism-Related Diseases, Annals of Internal Medicine, 140, 11, pp. 910-922.

Diggle, P.J., Rowlingsos, B., and T. Su (2004). Point Process Methodology for On-line Spatio-temporal Disease Surveillance, Johns Hopkins University, Department of Biostatistics Working Papers, paper 37.

Forsberg, L., Jeffery, C., Ozonoff, A., and M. Pagano (2006). A Spatiotemporal Analysis of Syndromic Data for Biosurveillance, Statistical Methods in Counterterrorism: Game Theory, Modeling, Syndromic Surveillance, and Biometric Authentication, A. Wilson, G. Wilson, and D.H. Olwell, eds., Springer, New York, NY, pp. 173-191.

Fricker, R.D., Jr. (2007). Syndromic Surveillance, Encyclopedia of Quantitative Risk Assessment (to appear).

Fricker, R.D., Jr., Knitt, M.C., and C.X. Hu (2007). Comparing Directionally Sensitive MCUSUM and MEWMA Procedures with Application to Biosurveillance, Quality Engineering (to appear).

Fricker, R.D., Jr., and H. Rolka (2006). Protecting Against Biological Terrorism: Statistical Issues in Electronic Biosurveillance, Chance, 91, pp. 4-13.

Fricker, R.D., Jr. (1997). Nonparametric Control Charts for Multivariate Data, Ph.D. Thesis, Yale University.

Kleinman, K., Lazarus, R., and R. Platt (2004). A Generalized Mixed Model Approach for Detecting Incident Clusters of Disease in Small Areas, with an Application to Biological Terrorism, American Journal of Epidemiology, 159, pp. 217-224.

Kulldorff, M. (2001). Prospective Time Periodic Geographical Disease Surveillance Using a Scan Statistic, Journal of the Royal Statistical Society, Series A (Statistics in Society), 164, pp. 61-72. Accessed online at www.satscan.org/papers/k-jrssa2001.pdf on November 28, 2006.

Kulldorff, M. (1997). A Spatial Scan Statistic, Communications in Statistics, Theory and Methods, 26, pp. 1481-1496. Accessed online at www.satscan.org/papers/k-cstm1997.pdf on November 28, 2006.

Lazarus, R., Kleinman, K., Dashevsky, I., Adams, C., Kludt, P., DeMaria, Jr., A., and R. Platt (2002). Use of Automated Ambulatory-Care Encounter Records for Detection of Acute Illness Clusters, Including Potential Bioterrorism Events, Emerging Infectious Diseases, 8, pp. 753-760. Accessed online at www.medscape.com/ viewarticle/440756_print on November 28, 2006. 
Lawson, A.B., and K. Kleinman (eds.) (2005). Spatial and Syndromic Surveillance for Public Health, John Wiley \& Sons.

Mandl, K.D., Overhage, J.M., Wagner, M.W., Lober, W.B., Sebastiani, P., Mostashari, F., Pavlin, J.A., Gesteland, P.H., Treadwell, T., Koski, E., Hutwagner, L., Buckeridge, D.L., Aller, R.D., S. Grannis (2004). Implementing Syndromic Surveillance: A Practical Guide Informed by the Early Experience, The Journal of the American Medical Informatics Association, 11, pp. 141-150. Accessed online at www.pubmedcentral.nih.gov/ articlerender.fcgi?artid=353021 on November 28, 2006.

Montgomery, D.C. (2001). Introduction to Statistical Quality Control, 4th edition, John Wiley \& Sons, New York.

Olson, K.L., Bonetti, M., Pagano, M., and K.D. Mandl (2005). Real Time Spatial Cluster Detection Using Interpoint Distances Among Precise Patient Locations, BMC Medical Informatics and Decision Making, 5. Accessed online at www.biomedcentral.com/1472-6947/5/19 on December 4, 2006.

Randles, R.H. and Wolfe, D.A. (1979). Introduction to the Theory of Nonparametric Statistics, John Wiley \& Sons, New York, New York.

Rogerson, P.A., and I. Yamada (2004). Monitoring Change in Spatial Patterns of Disease: Comparing Univariate and Multivariate Cumulative Sum Approaches, Statistics in Medicine, 23, pp. 2195-2214.

Shmueli, G., and S.E. Fienberg (2006). Current and Potential Statistical Methods for Monitoring Multiple Data Streams for Biosurveillance, Statistical Methods in Counterterrorism: Game Theory, Modeling, Syndromic Surveillance, and Biometric Authentication, A. Wilson, G. Wilson, and D.H. Olwell, eds., Springer, New York, NY, pp. 109-140.

Shmueli, G. (2006). Statistical Challenges in Modern Biosurveillance, in submission to Technometrics, draft dated September 18, 2006.

Sonesson, C. (2007). A CUSUM Framework for Detection of Space-time Disease Clusters using Scan Statistics, Statistics in Medicine (in press).

Sosin, D. (2005). Evaluation Challenges for Syndromic Surveillance - Making Incremental Progress, Morbidity and Mortality Weekly Report, 53 (Supplemental), Centers for Disease Control and Prevention, pp. $125-129$.

Sosin, D.M. (2003). Syndromic Surveillance: The Case for Skillful Investment View, Biosecurity and Bioterrorism: Biodefense Strategy, Practice, and Science, 1, 247-253. Accessed online at www.medscape.com/ viewarticle/466780 on November 22, 2006.

Waller, L.A., and C.A. Gotway (2004). Applied Spatial Statistics for Public Health Data, John Wiley \& Sons.

Woodall, W.H., Marshall, J.B., Joner, M.D., Jr., Fraker, S.E., and A.G. Abdel-Salam (2007). On the Use of Scan Methods in Prospective Public Health Surveillance, to be submitted to Journal of the Royal Statistical Society, Series A (Statistics in Society). Draft dated March 8, 2007.

Woodall, W.H. (2006). The Use of Control Charts in Health-Care and Public-Health Surveillance, Journal of Quality Technology, 38, pp. 1-16. 